\title{
Modelo Didáctico para la Facultad de Ciencias Administrativas y Económicas de la Universidad Técnica del Norte en Ecuador
}

\author{
Omar Abreu, Miguel E. Naranjo, Bertha S. Rhea y Mónica C. Gallegos \\ Universidad Técnica del Norte, Campus. Avenida 17 de julio, 5-21, General José María Córdova, Código \\ postal 199, Ibarra, Ecuador. (e-mail: oabreu@utn.edu.ec, menaranjo@utn.edu.ec, srheag@utn.edu.ec, \\ mgallegos@utn.edu.ec
}

Recibido Dic. 7, 2015; Aceptado Feb. 11, 2016; Versión final Mar. 7, 2016, Publicado Ago. 2016

\begin{abstract}
Resumen
Se presentan los resultados de un proyecto de investigación aplicado en la Facultad de Ciencias Administrativas y Económicas (FACAE) de la Universidad Técnica del Norte, en Ecuador. El objetivo fue diagnosticar la didáctica de los docentes de la facultad y proponer cambios y mejoras. La composición del cuerpo docente es muy diversa y heterogénea y no cuenta con la experiencia y la preparación didáctica suficientes para responder a las demandas de la docencia universitaria de estos tiempos. El modelo permitió diagnosticar los conocimientos didácticos de los profesores y la evaluación de los resultados de la aplicación del modelo didáctico mostro un mejor desempeño de los docentes de la FACAE y mejor comprensión del proceso de enseñanza-aprendizaje.
\end{abstract}

\section{A New Teaching Model for the Faculty of Management and Economics at the Technical University of the North, in Ecuador}

\begin{abstract}
The article is the result of a research project applied to the Management and Economic Sciences Faculty (FACAE) of the Technical University of the North, in Ecuador. The objective was to know and analyze the didactics of the faculty professors and propose changes and ways to improve. The composition of the professor of the faculty is varied and heterogeneous and there is not enough experience and didactic preparation to fulfill the present university teaching demands. The model allowed diagnosing the didactic knowledge of the professors and the evaluation of the results of the application of the didactic model showed better performance of the professors of the FACAE and a better comprehension of the teaching-learning process.
\end{abstract}

Keywords: didactic model; teaching-learning process; professor; student; organization forms 


\section{INTRODUCCIÓN}

La educación es un instrumento de cambio, un recurso salvador, una respuesta al estado mundial indeseado. Las universidades, convertidas en verdaderas casas de razón, son un destacamento de vanguardia en esa noble y justa aspiración humana que pueden hacer de la educación una fuerza para el avance social, la generación de riqueza, el fortalecimiento de las identidades, la cohesión social, la lucha contra la pobreza y la promoción de una cultura de paz; orientar la educación superior hacia la formación de personas capaces de enfrentar con ética, social y ambiental los retos en el desarrollo endógeno y la integración de países y participar activa, crítica y constructivamente en la sociedad de conocimientos, cambiar las formas de acceder, construir, producir, transmitir, distribuir y utilizar el conocimiento" (Bello, 2011). De la excelencia del claustro universitario depende el éxito de la misión de esta institución "(...) una formación de calidad apunta no sólo a la profesionalización en una carrera universitaria, sino también a una clara formación humana y creativa que permita enfrentar con solvencia los retos de la vida" (Rocha, 2013).

La formación de profesores concentra la atención de números estudiosos que insisten en las características de esta profesión. Para Castellanos, et .al., (2001) preparar a los estudiantes para explotar las potencialidades de la sociedad del conocimiento, es un desafío para los profesores. Fernández, (2010) resalta el valor profesional del educador y su relación con el desarrollo social, como responsables de un compromiso superior que consiste en formar integralmente a sus verdaderos hacedores. El profesor no solo participa en el complejo y dinámico proceso de formación de los protagonistas del desarrollo social. Su emprendimiento es superior. Él orienta, guía, conduce, dirige ese proceso, cuyos planes, dirección y control les corresponden. (Addine, et. al. 2004). Los recursos profesionales del profesor universitario deben ser abundantes, variados, eficaces y eficientes, pues se desenvuelve en un contexto cuyas metas lo complejizan le exigen un carácter multifuncional y una evaluación multifactorial.

En la Facultad de Ciencias Administrativas y Económicas (FACAE), de la Universidad Técnica del Norte, de la República del Ecuador, se ha creado un Modelo Didáctico para responder a esas complejidades, estableciendo la dialéctica profesor, estudiante, aprendizaje. Es una representación teórica abstraída de la práctica, de una parte de la realidad, cuya complejidad para ser estudiada y comprendida lo amerita. Sienta pautas en la solución de un problema y sirve de referencia, de objeto de reproducción, de aplicación y de imitación. El Modelo Didáctico de la FACAE es una construcción general sustentada en la teoría, la práctica educativa, la investigación y el entorno, para representar el funcionamiento del proceso de enseñanzaaprendizaje (PEA), en función de la formación integral de los estudiantes, en correspondencia con las demandas de desarrollo de la sociedad. Es un sistema concebido mentalmente, refleja o reproduce al PEA de cada tema estudio con la introducción sistemática de nuevos contenidos, la modificación renovadora del sistema de conocimientos de las asignaturas y el rediseño de los componentes didácticos del proceso para su estudio. Se estructuró a partir del modelo del profesional de las carreras. Tiene carácter sintético e intensivo pues no describe una estructura concreta del PEA, sino que se aparta de la realidad perceptible mediante un proceso de abstracción y subraya el atributo que adquieren las asignaturas con la introducción del nuevo contenido y las relaciones que se establecen entre este y el resto de los componentes: objetivo-método, medios de enseñanza-formas organizativas-sistema de evaluación, a partir de ello.

Posee las siguientes características: expresa las relaciones entre sus componentes, conserva el criterio del objetivo como categoría rectora y resalta el carácter dialéctico de sus relaciones con el contenido. Es guía para la introducción de nuevos contenidos de todas las asignaturas, a partir de la modificación del conocimiento y referencia para otras educaciones. Es abierto, susceptible de perfeccionamiento y enriquecimiento a partir de su introducción y generalización en la práctica, toma como punto de partida el diagnóstico integral y contextualizado de la realidad. Se aproxima al funcionamiento real del objeto (validez y confiabilidad). Incluye los cambios de la realidad (flexibilidad, utilidad y permanencia). Tiene carácter dinámico, se retroalimenta constantemente de la práctica, se aotoperfecciona. Entre sus componentes didácticos se expresa una dialéctica interna, su sistema de evaluación es un criterio de actualización del diagnóstico, que sirve de punto de partida para su remodelación. Tiene vigencia y necesidad en la sociedad ecuatoriana actual (contextualizado) y como pilares la relación entre la enseñanza y el aprendizaje, resultante de la impartición de las asignaturas y la relación dinámica entre todos los componentes del PEA. Se concibió a partir del modelo del profesional, se basa en el desarrollo de competencias docentes que integran el contenido del modo de actuación y se manifiestan en la idoneidad para el cumplimiento de las funciones que le son inherentes y exigidas, controladas y evaluadas a los docentes, por la sociedad.

En torno a la didáctica se ha desarrollado una polémica todavía vigente. La Didáctica está muy ligada a la Pedagogía, pero es una ciencia que tiene su objeto de estudio y su sistema teórico. Alcanza la categoría de ciencia al estudiar a un nivel cualitativo la organización y desarrollo del PEA, sus peculiaridades y la relación educador-alumno mediada por los: objetivos, contenidos, métodos, formas, medios, evaluación desde un objeto preciso del conocimiento (Pla, et al., 2010). Se ocupa de los problemas comunes a las asignaturas, 
abstrayéndose de las particularidades de una asignatura, dentro del (PEA), dimensión esencial y núcleo del proceso educativo. La Didáctica: es el arte transmitir conocimientos de forma más adecuada. Sistematiza e integra aspectos teóricos y metodológicos del proceso de comunicación y lo enriquece. Cobija las teorías, métodos y estrategias aplicadas al PEA. Existe para quienes se dedican a la formación de estudiantes y tienen criterios para planear, organizar, desarrollar, ejecutar y evaluar los procesos del aprendizaje. Hace más consciente y eficiente la acción del profesor y más interesantes y provechosos los estudios del alumno. Propicia que la institución educativa se convierta en un lugar de razonamiento activo, dinámico, de trabajo, de creación y desarrollo según el proyecto educativo que asume. Organiza y estimula el proceso de reflexión y acción del docente y de sus discípulos.

\section{METODOLOGÍA}

El equipo de investigadores hizo coincidir población y muestra considerando que la facultad cuenta con 127 docentes y que existen facilidades para trabajar con todos. Se diseñaron y aplicaron 7 instrumentos para recoger información relacionada con la formación didáctica, el dominio de esta ciencia, el interés y la motivación por conocer su teoría y la aplicación de ella en el ejercicio docente. Se utilizó el método históricológico para conocer la evolución del proceso de Formación Didáctica de los docentes de la FACAE. EI sistémico para diseñar el nuevo Modelo Didáctico con carácter integrador y para elaborar la guía que se aplicó en la observación de clases. La modelación para representar el proceso de Formación Didáctica. El analítico-sintético para la interpretación de la información aportada por los métodos y técnicas que sirvieron para el diagnóstico y el inductivo-deductivo para determinar las potencialidades y las limitaciones del sistema Formación Didáctica de los docentes y realizar la propuesta del nuevo Modelo Didáctico. Se realizó el análisis documental para el estudio de la documentación relacionada con los antecedentes del proceso de Formación Didáctica de los docentes y el contenido del nuevo Modelo Didáctico. Se aplicaron encuestas a estudiantes, profesores y directivos para recoger información acerca del impacto trasformador del nuevo Modelo Didáctico y la triangulación de fuentes de información para garantizar la confiabilidad de los resultados en la evaluación del nuevo Modelo Didáctico. Se observaron clases aplicando una guía con dimensiones e indicadores forman parte del nuevo Modelo Didáctico y para evaluar el ascenso de la calidad de la docencia durante la puesta en práctica del mismo.

Por calidad del modelo didáctico se asume su rigor científico y metodológico, en correspondencia con criterios relacionados con: posibilidades de utilización práctica, actualidad, fiabilidad, novedad y significación práctica. Para evaluar la calidad se utilizó la metodología de la comparación por pares del criterio de expertos. Se seleccionaron 5 expertos ecuatorianos, 3 cubanos, un boliviano, 1 argentino, 1 español y 1 colombiano. Los indicadores para la evaluación fueron: Muy Adecuado, tres lo evaluaron así, Bastante Adecuado, para 6, Adecuado, 2 coincidieron con este criterio, Poco Adecuado y No Adecuado.

\section{EL MODELO DIDÁCTICO: SUS COMPONENTES}

EI PEA universitario es un sistema en el que prevalece un contrapunteo y una tensión dialéctica vigentes entre las tendencias conservadoras y reproductoras (Mayorga, J y Madrid, D. 2010), que son dominantes, y las tendencias transformadoras que propugnan otro modelo de desarrollo humano (García, 2000).

Tabla. 1. Modelo didácticos

\begin{tabular}{|l|l|l|l|}
\hline \multicolumn{1}{|c|}{ Tradicional } & \multicolumn{1}{c|}{ Tecnológico } & \multicolumn{1}{c|}{ Esponteneísta-activista } & \multicolumn{1}{c|}{ Alternativo } \\
\hline Es el más persistente. & Tiene un enfoque & Centra su atención en el & Defiende el enriquecimiento \\
Trata el contenido & positivista. Sobre & interés y el convencimiento & progresivo del conocimiento. \\
enciclopédicamente, de & dimensiona los & para dominar el contenido & Integra diverso referentes. Exige \\
manera fragmentada y & objetivos. Se atribuye & de la realidad que rodea al & modelos complejos de entender \\
con enfoque disciplinar. & el rigor y la eficiencia. & estudiante. & el mundo y de actuar en él. \\
\hline
\end{tabular}

El Modelo Didáctico de la FACAE se fundamenta en el Enfoque Histórico Cultural de Vygotsky. Asume el PEA con un enfoque desarrollador, integrador y contextualizado de la Didáctica. Responde a las demandas y problemas sociales expresados en sus objetivos. Parte del diagnóstico para concebir el PEA y atender al desarrollo desde las potencialidades. Contextualiza la educación, en el PEA y a la sociedad a través de las problemáticas como contenido de enseñanza para atender a los objetivos generales y ejes transversales desde las asignaturas y a las potencialidades, necesidades e intereses de los estudiantes. Contextualiza el PEA de la asignatura con la incorporación de componentes y contextos del proceso educativo de la universidad, la familia y la comunidad a través de la selección y utilización de fuentes diversas de información y de métodos participativos de búsqueda y solución de problemas investigativos. Integra los contenidos de 
la asignatura a través de la sistematización del conocimiento de la ciencia en la familiarización, comprensión, dominio, sistematización, autoevaluación y en la asimilación consciente del contenido, a nivel de tarea, clase, unidad, y programa y en una concepción interdisciplinaria en el tratamiento de conocimientos, habilidades, valores, métodos y evaluación. Armoniza la educación atendiendo a lo cognoscitivo y a lo actitudinal en el diagnóstico, los objetivos, los contenidos, los métodos, y la evaluación logrando el vínculo con la vida, con la práctica, con el trabajo y aporta a la Pedagogía y a la Didáctica la idea de un proceso educativo caracterizado por la actividad y la comunicación desarrolladoras de quienes se educan, motivados por problemáticas del contexto socio histórico (Pla, et al., 2010).

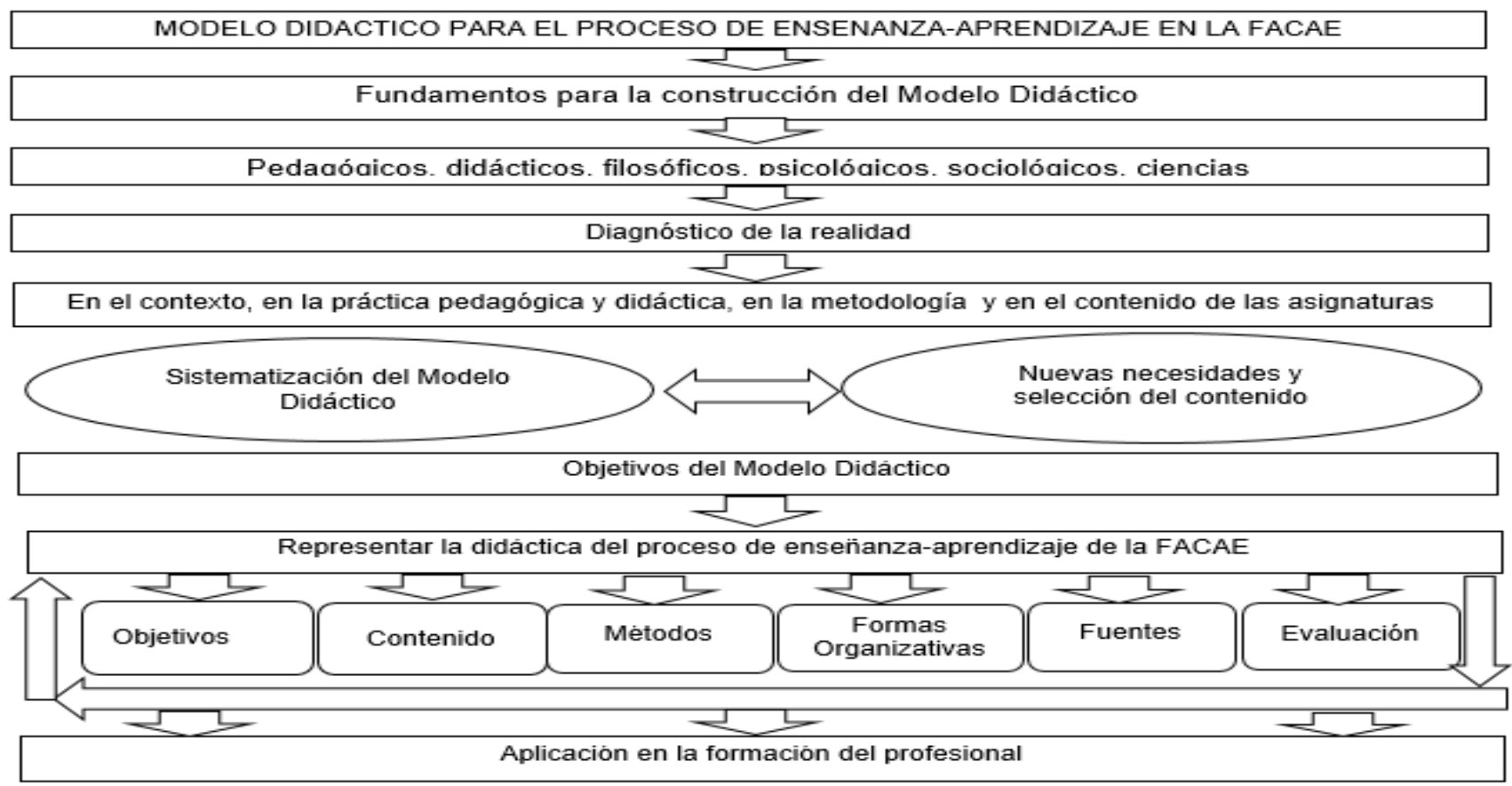

Fig.1. Representación del Modelo Didáctico de la FACAE

\section{El Diagnóstico}

El $37.5 \%$ de los profesores de la FACAE manifestó no haber recibido nuca curso de Pedagogía, el $53.8 \%$ no los ha recibido de Didáctica y el $65 \%$ no se ha capacitado en la metodología de sus asignaturas. El $33 \%$ desconocía el objeto de estudio de la Didáctica, el $21.4 \%$ no tenía en sus prioridades dominar el contenido de esta ciencia, ni le concedía importancia. El 34.2 no la aplicaba en sus clases y el 72.45 reconoció poseer un dominio medio y bajo de la Didáctica.

Tabla.2. Comparación entre el conocimiento de la asignatura que imparten los docentes y la Didáctica

\begin{tabular}{|c|c|c|c|c|}
\hline Orden & Índice de conocimiento de la signatura que imparte & $\%$ & Índice de conocimiento de la Didáctica & $\%$ \\
\hline 1 & 10 & 33 & 5 & 23.6 \\
\hline 2 & 9 & 29.2 & 8 & 21.7 \\
\hline 3 & 8 & 20.8 & 7 & 19.8 \\
\hline 4 & 5 & 5.7 & 10 & 11.3 \\
\hline 5 & 7 & 4.7 & 9 & 8.5 \\
\hline 6 & 6 & 1.9 & 4 & 6.6 \\
\hline 7 & 4 & 1.9 & 3 & 5.7 \\
\hline 8 & 3 & 0.9 & 6 & 1.9 \\
\hline 9 & 2 & 0.9 & 2 & 0.9 \\
\hline 10 & 1 & 0.9 & 1 & 0 \\
\hline 11 & 0 & 0.9 & 0 & 0 \\
\hline
\end{tabular}

En las clases que se visitaron se constataron las mayores deficiencias en la comprensión de los perfiles del docente y los estudiantes, en el empleo de formas organizativas inapropiadas para el tratamiento algunos contenidos, en la concepción del aprendizaje y en la concepción y utilización de la evaluación. 


\section{La relación profesores estudiantes}

En el PEA, intervienen dos componentes personales del proceso educativo, que son los más importantes e imprescindibles: el estudiante y el profesor (Moreno et, al 2014), La formación de los docentes en un proceso permanente, continuo, organizado y sistémico e integral, es una necesidad para lograr la formación del estudiante como el profesional que demanda la sociedad (Acosta, et al., 2015).

\section{Los docentes}

El profesor como componente personal del proceso educativo que asume la condición de sujeto cuando logra la preparación, para diseñar, orientar, controlar y evaluar, la educación de los estudiantes, crea un ambiente educativo en todos los contextos y perfecciona su actividad con la investigación (Pla, et al., 2010). En el Modelo Didáctico se define al docente como una persona cabal y del momento histórico que le ha correspondido, consciente de que ningún tiempo es mejor que otro, no puede vivir al margen de las masas, aislado de su sociedad, de sus objetivos, de sus sueños, de sus aspiraciones, de sus proyectos y de su realidad: debe ser un protagonista activo de esa dinámica constructiva y debe estar identificado con las aspiraciones más sagradas de su pueblo. La Universidad demanda el desvelo de este profesional por la sabiduría plena y placentera, la inconformidad constante con las verdades exclusivas, absolutas y acabadas, con las respuestas únicas e irreflexivas y los puntos de vistas unilaterales, sobre un contenido determinado.

La sensibilidad es un rasgo del profesor universitario. La sabiduría no puede venirle únicamente del estudio pasivo, plano y lineal de los pensadores, debe ser alimentada también, por el aliento de los protagonistas de la historia, de todos, grandes y humildes, no solo de sus héroes más legendarios. Debe ser capaz de transmitir y formar sentimientos. El sentido de ejemplaridad profesional también debe caracterizarlo, como un modelo de amplio dominio del contenido, con enfoque universal. No se trata solo del contenido de su asignatura, sino del andamiaje teórico y práctico de las ciencias que intervienen en ese complejo proceso de formación del ser humano, del arte de transmitir lo que se conoce, con sentido utilitario. El docente debe ser capaz de colocarse en el lugar de sus estudiantes, de conocer de ellos todo lo que su profesionalidad le permita, de meditar sobre las manifestaciones conductuales de estos, comprenderlos en toda su diversidad y de utilizar esa información para facilitar el desarrollo de sus potencialidades.

Como investigador consecuente debe lograr un modo de actuación que lo revele como profesional del currículo, como un investigador del proceso que dirige sobre bases científicas, que lo armoniza, lo sistematiza, lo valida en la práctica pedagógica revolucionaria y patriótica, lo rediseña a partir de las nuevas exigencias históricas y sociales, y lo asume como un proyecto o modelo global que incluye la planificación, la organización, la ejecución y el control. Un proceso que tiene como centro la práctica, la realidad cambiante y dinámica, la vida en su más amplia y contradictoria diversidad, que implica a las instituciones y a los agentes educativos, especialmente a la escuela, a los docentes, a los estudiantes, a la familia, a la comunidad, y a la sociedad en su conjunto y tiene naturaleza dinámica, manifestación activa, flexibilidad objetiva, amplitud abierta y capacidad científicamente adaptable a las circunstancias histórico concretas.

El profesor es protagonista y el responsable de la enseñanza. Es un agente de cambio que participa desde sus saberes, en el enriquecimiento de los conocimientos y valores más preciados de la sociedad. Asume la dirección creadora del PEA, planificando y organizando la situación de aprendizaje, orientando a los estudiantes y evaluando el proceso y el resultado. La autoridad del profesor y su ascendencia sobre los estudiantes se sustenta en la demostración de una amplia cultura en la que se distinguen su sabiduría acerca de contenido de la asignatura y del arte para enseñarla bien: dígase la Metodología, la Didáctica y la Pedagogía. Es autoridad está asociada a una concepción humanista-dialéctica del PEA, que garantice que sus estudiantes enfrenten exitosamente todas las tareas de su aprendizaje por compleja que sean, bien planificadas y dosificadas y que en ese proceso se conviertan sistemáticamente en mejores seres humanos.

\section{Los estudiantes}

El estudiante es el componente personal del proceso educativo que asume la condición de sujeto del mismo en la medida que es consciente y protagonista de su educación. (Pla, et al., 2010). El Modelo Didáctico definió el perfil del estudiante en los términos siguientes. No puede ser simple colecto o receptor pasivo de conocimientos transmitidos por el profesor, auxiliado de todos los medios y las fuentes a su alcance. Debe conocer los objetivos de la sociedad en que se desarrollan y asumirlos con espíritu crítico y renovador. Demostrará una posición voluntariosa, entusiasta, de alta disposición y de elevado compromiso para el aprendizaje. Identificará la necesidad de apropiarse del nuevo contenido, de elevarlo a la categoría de conocimiento utilitario e incorporarlo a su modo de actuación. Tendrá una conciencia crítica en relación con su entorno, local, nacional e internacional y para descubrir la importancia del contenido que recibe y convertirlo en nuevo conocimiento, para transformar de ese escenario. Aceptará la conducción del profesor sin dejar de 
ser un componente activo del PEA, participando en diálogos, en discusiones académicas y en la solución de sus problemáticas. Asumiendo una actitud contextualizada y disfrutando a plenitud de las libertades creadoras y constructivas de su proceso formativo.

\section{Formas organizativas}

En el Modelo Didáctico se priorizan como formas organizativas la conferencia panorámica, la clase práctica, el seminario y los talleres integradores, aunque los docentes tienen la posibilidad de incluir otras. La conferencia panorámica se ofrecen a los estudiantes orientaciones para el estudio del tema sobre la base del diagnóstico, se conocen sus criterio sobre las propuestas, se realiza la presentación del tema, se orienta hacia el logro de los objetivos y se explica el sistema de contenidos, los métodos que se aplicarán, el sistema de evaluación y las fuentes que deben ser utilizadas. La clase es la organización del estudio del tema con la utilización de las fuentes orientadas por el profesor. En ella se confrontan puntos de vista y de autores de diferentes posiciones, incluyendo las notas de clases y los estudiantes realizan comparaciones, resúmenes, síntesis, mapas conceptuales, esquemas lógicos, ubican en mapas, resuelven problemas, realizan cálculos, preparan representaciones de la realidad de su futura profesión, y redactan ponencias.

El seminario se concibe como una profundización en el dominio del contenido, que exige el empleo de recursos comunicativos para su exposición. Estimula el crecimiento y la demostración de los estudiantes. Su organización puede ser en paneles, mesas redondas, debates, simposios, representaciones, ponencias y oponencias, diálogos, foros, discusiones y otras modalidades que posean una fundamentación didáctica. Los talleres son espacios para que los estudiantes trabajen en equipo con las fuentes del contenido bajo la conducción del profesor para el análisis, la síntesis, la integración, la presentación y el debate de la información recopilada y procesada para ejecutar las tareas orientadas previamente. Los talleres propician la profundización, la generalización, la integración y la aplicación del contenido a situaciones cognoscitivas y metacognoscitivas nuevas. En el taller integrador los estudiantes exponen todo el contenido relacionado con el un tema y lo vinculan con situaciones propias de su futura profesión. Demuestran los niveles de conocimientos y las habilidades desarrolladas en el PEA del tema, la aplicación de este contenido en su actuación como miembros activos de la comunidad en su gestión transformadora de la sociedad y el desarrollo de su capacidad comunicativa mediante la exposición de los resultados del proceso investigativo.

\section{El aprendizaje}

El Modelo Didáctico de la FACAE concibe un aprendizaje, óptimo, auténtico, significativo y desarrollador que implica una concepción moderna de la clase y una posición activa de los estudiantes sobre la base de la motivación, el interés, la disposición, la entrega, la autorregulación, la búsqueda, la indagación y el empleo de métodos nuevos, el alumno aprende resolviendo problemas nuevos para él, cuando dispone de conocimientos y habilidades para ello y aplican la creatividad (Álvarez, 1999). Para Castellanos et, al., (2001) el aprendizaje desarrollador promueve el desarrollo integral de la personalidad, la apropiación de conocimientos, destrezas y capacidades intelectuales en armonía con los sentimientos, motivaciones, cualidades, valores, convicciones e ideales. Potencia el tránsito progresivo de la dependencia a la independencia y a la autorregulación de la capacidad que le permite conocer, controlar y transformar creadoramente su propia personalidad y su medio. Permite realizar aprendizajes durante toda la vida, desarrollando habilidades y estrategias para aprender a aprender y la necesidad de la autoeducación.

Los estudiantes están aprendiendo cuando: hacen observaciones sobre hechos, procesos, películas y demostraciones; hacen planes y realizan experiencias, comprueban hipótesis y anotan sus resultados; consultan libros, revistas, diccionarios; toman apuntes y organizan ficheros y cuadros comparativos; escuchan, leen, anotan, pasan en limpio apuntes y los complementan con extractos de otras fuentes; formulan dudas, piden aclaraciones, suscitan objeciones, discuten, comparan y verifican; colaboran con el profesor y se auxilian en las tareas, en la aclaración de dudas y en la solución de problemas; efectúan cálculos y usan tablas; dibujan e ilustran; copian mapas, los reducen o amplían a escala; completan e ilustran mapas mudos, buscan, coleccionan, identifican, comparan y clasifican muestras, modelos, sellos, grabados, plantas, objetos, fotografías, responden a interrogatorios y tesis, procuran resolver problemas, identifican errores, corrigen los suyos propios o los de sus colegas (Addine, et al., 2004).

Aprenden cuando analizan y reflexionan responsabilidad acerca de la manera en que aprenden; de las estrategias que utilizan, de la calidad con que sus profesores conducen el PEA y de sus resultados. Cuando autorregulan ese proceso mediante estrategias que no son rígidas, ni dogmáticas, si no flexibles y adaptables al contexto en que se desarrollan. Cuando establecen un diálogo productivo y desarrollador entre los conocimientos previos, aquellos que poseen con anterioridad y logran mantener activos en sus estructuras mentales y los conocimientos que reciben en incorporan como nuevos y en ese proceso crean un conocimiento que también es novedoso, pero que resulta superior al que ellos poseían, que les permite enfrentar con éxito situaciones desconocidas y tiene una utilidad práctica para la vida. Cuando desarrollan la 
actividad creadora, componente que expresa la asimilación de criterios, métodos y procedimientos que permiten a los estudiantes enfrentar los problemas de la vida a través de soluciones creativas, novedosas y que generan un análisis autovalorativo de la estrategia de aprendizaje que utilizaron, de su actuación y de las vías empleadas en la solución de los problemas. Está asociado a niveles de integración, generalización, metacognición y contextualización del resto del contenido y constituye un aspecto decisivo en la formación de la personalidad que tiene que ser estimulado, conducido y controlado por el profesor.

\section{El sistema de evaluación}

La evaluación tiene funciones como diagnosticar, controlar, instruir, educar, estimular, orientar y desarrollar, es una actividad o proceso sistemático de identificación, recogida o tratamiento de datos sobre elementos o hechos educativos, con el objetivo de valorarlos de tomar decisiones (García y Pérez, 1989), que se relaciona con todos los componentes del PEA, pues todos sus momentos se evalúan. Se debe convenir con los estudiantes mediante indicadores definidos de manera conjunta. Coloca al estudiante en posición de éxito, no de fracaso y ante situaciones nuevas que generan contradicciones entre lo conocido y lo desconocido, lo viejo y lo nuevo, cuya solución provoca transformaciones en él, en sus conocimientos y en su modo de actuación. Incluye autoevaluación, coevaluación y heteroevaluación. No puede concebirse al margen de la diversidad, ni asume el error como expresión de incompetencia, sino como oportunidad para estimular el desarrollo. No se concibe como medio de coercitivo. Es un recurso didáctico al servicio del PEA. No se reduce a una expresión calificativa cuantitativa o cualitativa. Está orientada al mejoramiento del estudiante y de su proceso de aprendizaje y no es el fin del mismo, ni es un fin en sí misma.

Todas las actividades planificadas son evaluadas. El sistema de evaluación se concentra en la exigencia de demostraciones relacionadas con la búsqueda, el procesamiento y la utilización del contenido, en la ejecución de las tareas docentes, a partir del estudio de las fuentes indicadas por el profesor y otras localizadas por los estudiantes, la orientación, la motivación y el control del aprendizaje, la autorregulación de su propio aprendizaje, el desarrollo de la expresión oral y escrita, la capacidad para analizar, resumir y sintetizar, la emisión y argumentación de criterios propios y lógicos, la comunicación grupal, la condición de modelo de comunicador del profesor, en el uso del contenido del tema de estudio para lograr el protagonismo social, con la familia y la comunidad, en la planificación y en la comunicación de sus resultados y su introducción en la práctica para solucionar problemas que afectan a la sociedad.

\section{RESULTADOS}

El primero es la elaboración del Modelo y su puesta en práctica mediante el desarrollo de un curso de capacitación a los docentes de la FACE de 40 horas de duración, de reuniones metodológicas, talleres y clases demostrativas. Se evidenciaros cambios significativos el modo de actuación de los docentes, la relación entre el índice de conocimientos de las asignaturas impartidas y la didáctica se equilibró, (Tabla. 2).

Tabla.3. Índice de conocimiento de la Didáctica después de la puesta en práctica del modelo

\begin{tabular}{|c|c|c|}
\hline Orden & Índice de conocimiento de la Didáctica & $\%$ \\
\hline 1 & 8 & 27.5 \\
\hline 2 & 10 & 23.5 \\
\hline 3 & 9 & 15.7 \\
\hline & & \\
\hline 4 & 7 & 7.8 \\
\hline 5 & 6 & 2 \\
\hline 6 & 5 & 2 \\
\hline 7 & 4 & 2 \\
\hline
\end{tabular}

El 98.5\% del claustro reconoció la necesidad del Modelo y declaró que modificaría su modo de actuación, el $78 \%$ lo evaluó como excelente, el 20.45 como bueno y el 1.9\% como regular. El 93\% aceptó su utilidad. Para el $98 \%$ dominar el contenido de la Didáctica es de alta prioridad. El $96 \%$ reconoce que tiene una importancia alta para concebir e impartir sus clases. El $91.8 \%$ ubica su dominio actual entre los niveles alto y medio, $34.7 \%$ alto y $57.1 \%$ medio. El $80 \%$ reconoce en el PEA al objeto de estudio de esta ciencia.

\section{CONCLUSIONES}

El diagnóstico de los docentes de la FACAE de la UTN, de la Repúblico del Ecuador, evidenció el desconocimiento de la Didáctica y la necesidad de aplicar un modelo didáctico que permita la transformación del modo de actuación de los docentes para conducir el PEA de sus estudiantes en función del aprendizaje desarrollador. El Modelo Didáctico de la FACAE se concibió como resultado de la integración de la investigación universitaria, el desarrollo de la práctica educativa universitaria, del ejercicio de la docencia y 
del proceso de retroalimentación sistemática con el entorno de la Universidad en relación con las demandas establecidas para la formación de sus profesionales.

La aplicación de Modelo puso reveló cambios en el modo de actuación de los docentes en el desempeño para diseñar, orientar, motivar, conducir y controlar el aprendizaje activo y desarrollador, para la creación de condiciones para el desarrollo de relaciones en un clima favorable, como modelo de comunicador, en su expresión oral, escrita y corporal, en relación con el uso del contenido de la asignatura como elemento integrador de los compontes del Modelo, objetivo, método, medios, formas organizativas, fuentes y evaluación, en la planificación de la investigación del PEA, en el procesamiento científico de la información obtenida y en la comunicación e introducción de los resultados investigativos en la transformación del PEA.

\section{REFERENCIAS}

Acosta, L.A, Abreu, O, Coronel, M. Sistema de Formación Pedagógica en la Universidad de Otavalo en Ecuador, DOI: 10.4067/S0718-50062015000200007. Revista, Formación Universitaria (en línea) 8 (2), 43-52 (2015)

Addine, F y otros 9 autores. Didáctica Teoría y Práctica., 1ra Edición, Pueblo y Educación, 1-291, La Habana, Cuba (2004)

Álvarez, C. La escuela en la vida. 2da Edición, Pueblo y Educación, 1-227, La Habana, Cuba (1999)

Bello, J. R. Principios y conceptos referidos a las universidades. http://bibliobytes.unimet.edu.ve, ISSN: 16908791, Revista Cuadernos Unimetanos, 25 (1) (en línea) 1-7 (2011)

Castellanos, D. Castellanos, B. Llivina, M. J. y Silverio, M. Hacia una concepción del aprendizaje desarrollador, 1ra Edición, Colección Proyectos, Instituto Superior Pedagógico E. J. Varona, 1-96, La Habana, Cuba (2011)

Fernández, M. M. Propuesta de formación pedagógica del profesorado de Educación Superior: Fundación tecnológica Antonio de Arévalo, 1ra Edición, Universidad Complutense de Madrid, 1-249, Madrid, España (2010)

García, J.M y Pérez, R. Diagnóstico, evaluación y toma de decisiones. 1era Edición, Rialp, 1-123, Madrid, España (1989)

García, F.F. los modelos didácticos como instrumento de análisis y de intervención en la realidad educativa. ISSN 1138-9796. Revista, Bibliográfica de Geografía y Ciencias Sociales (en línea) 207 (5), 55-78 (2000)

Mayorga M.J. y Madrid, D. Modelos didáctico y Estrategias de enseñanza en el Espacio Europeo de Educación Superior, ISSN 1989-8614. Revista. Tendencias Pedagógicas, 15 (1), 91-111 (2010)

Moreno, C., Molina, Y. y Chacón, J. Impacto del estilo pedagógico integrador en los estudiantes de licenciatura en Educación Básica de la Facultad de Estudios a Distancia, DOI: 10.4067/S071850062014000600005. Revista, Formación Universitaria, (en línea), 7 (6), 37-44 (2014)

Pla, R. y otros 16 autores. Una concepción de la Pedagogía como ciencia desde un enfoque histórico cultural. 1ra Edición, Pueblo y Educación, 1-79, La Habana, Cuba (2010)

Rocha, R. Escala de opinión de los estudiantes sobre la efectividad de la docencia (EOEED) en Educación Superior. DOI. 10.4067/S0718-50062013000600003. Revista, Formación Universitaria, (en línea), 6 (6), 13$22(2013)$ 